\title{
LAS ACTIVIDADES DE LA VIDA DIARIA EN PERSONAS MAYORES DURANTE EL CONFINAMIENTO
}

\author{
Jesús González-Moreno \\ Universidad Internacional de Valencia \\ jesus.gonzalezm@campusviu.es \\ Francisco Javier Román González \\ Universidad Autónoma de Madrid \\ María Cantero García \\ Universidad Internacional de Valencia
}

Recepción Artículo: 16 mayo 2021

Admisión Evaluación: 16 mayo 2021

Informe Evaluador 1: 22 mayo 2021

Informe Evaluador 2: 25 mayo 2021

Aprobación Publicación: 01 junio 2021

\section{RESUMEN}

El estado de alarma declarado por el Gobierno de España durante la pandemia de la COVID-19, ha afectado en gran medida al estado y a las actividades de la vida diaria de una población especialmente vulnerable son las personas mayores. El objetivo de este estudio es explorar el impacto de la pandemia en las personas mayores en cuanto a sus actividades de la vida diaria y su relación con el estado emocional. Se realizó un estudio de tipo expostfacto, con finalidad descriptiva y exploratoria, con un único tiempo de media (transversal). La recogida de datos se realizó mediante encuestas online destinadas a personas mayores de 50 años españolas, donde se incluyó la valoración de sociodemográficos, junto con aspectos específicos de la pandemia y una escala sobre actividades de la vida diaria realizada ad-hoc basada en instrumentos validados. Los resultados ponen de manifiesto que las AVD se han visto afectadas durante el confinamiento en personas de mediana y avanzada edad, especialmente las ABVD. Se ha encontrado una relación entre mantener las ABVD y un mejor estado anímico, así como una relación entre edad y mantener ese tipo de actividades

Palabras clave: COVID-19; personas mayores; actividades de la vida diaria

\section{ABSTRACT}

Activities of daily living in the elderly during confinement. The state of alarm declared by the Spanish Government during the COVID-19 pandemic has greatly affected the state and activities of daily living of a particularly vulnerable population, the elderly. The aim of this study is to explore the impact of the pandemic on the elderly in terms of their activities of daily living and their relationship with emotional state. An exploratory, descriptive, descriptive and exploratory study was carried out with a single mean time (cross-sectional). Data collection was carried out by means of online surveys aimed at people over 50 years of age in Spain, which inclu- 


\section{LAS ACTIVIDADES DE LA VIDA DIARIA EN PERSONAS MAYORES DURANTE EL CONFINAMIENTO}

ded the assessment of sociodemographic data, together with specific aspects of the pandemic and a scale on activities of daily living carried out ad-hoc based on validated instruments. The results show that ADLs have been affected during confinement in middle-aged and elderly people, especially ADLS. A relationship was found between maintaining ADLs and a better mood, as well as a relationship between age and maintaining these activities.

Keywords: COVID-19; elderly people; activities of daily living

\section{INTRODUCCIÓN}

El 11 de marzo de 2020 la Organización Mundial de la Salud (OMS) declaró la COVID-19 como pandemia mundial. Además de su elevada morbilidad y mortalidad, las repercusiones económicas están siendo enormemente graves. El Gobierno de España aprobó un Real Decreto (RD 463/2020, de 14 de marzo) por el que se declaraba el estado de alarma para la gestión de la situación de crisis sanitaria ocasionada por la COVID-19, cuyo objetivo era proteger la salud de la ciudadanía, contener la progresión de la enfermedad y reforzar el sistema de salud pública.

Una de las consecuencias que tuvo la declaración de la pandemia fue el confinamiento en el hogar, situación sin precedentes recientes en nuestro país, y con un importante impacto en el bienestar físico y psicológico de toda la población, especialmente de las personas mayores. La paralización de la actividad económica, el cierre de centros educativos y el confinamiento de toda la población durante semanas ha supuesto una situación extraordinaria y con múltiples estímulos generadores de estrés. Durante el confinamiento, los dos factores que más afectan al bienestar físico y psicológico son la pérdida de hábitos y rutinas y el estrés psicosocial, de acuerdo al primer estudio que analiza el impacto psicológico de la cuarentena por COVID-19 en China (Wang, Pan et al., 2020). La interrupción de hábitos durante el confinamiento y la instauración de otros poco saludables (p.ej. malos hábitos alimenticios, patrones de sueño irregulares, sedentarismo y mayor uso de las pantallas) pueden derivar en problemas físicos 0 en alteraciones en las actividades de la vida diaria.

Las actividades de la vida diaria (AVD) son el conjunto de aquellas actividades típicas que una persona realiza diariamente 0 asiduamente, y que permiten vivir de forma autónoma e integrada en la sociedad, cumpliendo así su rol dentro de ella (Costa et al., 2006; Reed y Sanderson, 1980). Dentro de estas podemos diferencian distintos tipos tres según la cantidad de recursos personales que impliquen: En primer lugar, se encuentran las actividades básicas de la vida diaria (ABVD) implican aquellas actividades rutinarias de autocuidado esencial (comer, beber, vestirse, dormir...). En segundo lugar, encontraríamos las actividades instrumentales de la vida diaria (AIVD) que comprenden

actividades más complejas que tienen como objetivo la interacción con el entorno (cocinar, comprar, gestionar dinero, cuidar... .). Por último, encontraríamos las actividades avanzadas de la vida diaria (AAVD) incluye actividades que posibilitan el desarrollo personal del sujeto dentro de la sociedad, requieren organización y mayor dificultad, y no son indispensables para la supervivencia (Días et al.,2015; Romero,2007).

Una mayor capacidad para poder llevar a cabo este tipo de actividades ha sido considerada como una medida del funcionamiento/independencia de las personas (especialmente las ABVD y las AIVD) y su ejecución está estrechamente ligada con la esperanza de vida libre de discapacidad (Escobar el al., 2012). Además, la realización o mantenimiento de las AVD se ha relacionado de forma positiva con bienestar psicológico en personas mayores (Meléndez et al., 2011) y se ha encontrado una relación moderada entre realizar un mayor número de actividades y tener mayor nivel de salud (Aguilar-Parra et al., 2016). En definitiva, las AVD resultan un aspecto importante a tener en cuenta en el envejecimiento, mantener y fomentar su realización para fomentar la independencia funcional de las personas y mejorar su calidad de vida.

La realización de las AVD se puede ver afectada por enfermedades que tienden a ocurrir con mayor probabilidad durante la vejez como pueden ser las demencias, donde desde prácticamente fases iniciales se encuentran problemas en la ejecución de AIVD, siendo este un posible indicativo de diagnóstico final confirmatorio (Álvarez et al., 2011). Como es lógico también se ha encontrado que problemas en el aparato locomotor en personas 
mayores comprometen de manera importante el desempeño de AVD, afectando de forma importante su calidad de vida (Veiguela et al., 2018). Además, se ha encontrado que enfermedades crónicas como la hipertensión, problemas circulatorios, reumatismo 0 enfermedad mental afectan a la capacidad deterioro funcional para las actividades de la vida diaria (Rubio et al., 2009). Otro aspecto no tan prevalente en personas mayores, pero que sí que tiene una elevada repercusión sobre la funcionalidad de la persona y una reducción de la participación en actividades de la vida diaria y de interés es la sintomatología depresiva (Aravena, 2017).

La situación de pandemia y confinamiento provocado por el coronavirus SARS-CoV-2

supuso un cambio en la rutina diaria, conllevando niveles de estrés, ansiedad, tristeza

y sentimientos de aislamiento, así como, implicó la adaptación a nuevas realidades cambiantes (Horesh y Brown, 2020; Ozamiz-Etxebarria et al., 2020). En este contexto, la población mayor se vió especialmente afectada por los efectos de la pandemia (Petretto y Pili, 2020). No obstante, parece que la respuesta emocional de las personas mayores fue relativamente baja y que no impactó de forma importante en su vida diaria (FernándezBallesteros y Sánchez-Izquierdo, 2020). De hecho, en algunos trabajos se afirma que las personas mayores informaron de menor sintomatología ansiosa y depresiva que los de mediana edad y que la población joven (LosadaBaltar, 2020). Una posible explicación a esto podría venir definida por el un mantenimiento en la realización de las AVD, aspecto que como se ha visto es importante en las personas mayores.).

Por tanto, resultaría interesante poder valorar la emisión y realización de las AVD en un contexto de limitación de libertad y confinamiento como el provocado por la pandemia derivada del COVID-19, especialmente en personas mayores por la implicación que el mantenimiento de la actividad tiene para pronosticar una mejor salud y bienestar (Fernández, 2002).

\section{OBJETIVOS}

Explorar el impacto de la pandemia en las personas mayores en cuanto a sus actividades de la vida diaria y su relación con el estado emocional.

\section{MATERIAL Y MÉTODO}

Se realizó un estudio de tipo expostfacto, con finalidad descriptiva y exploratoria, con un único tiempo de media (transversal).

La recogida de datos se realizó mediante encuestas online destinadas a personas mayores de 50 años españolas, donde se incluyó la valoración de sociodemográficos, junto con aspectos específicos de la pandemia y una escala sobre actividades de la vida diaria realizada ad-hoc basada en instrumentos validados como se verá posteriormente. Por último, se incluyo una escala de valoración del estado de ánimo.

La encuesta se distribuyó entre contactos y mediante redes sociales, y se invitó a los participantes a que remitieran a otros contactos. Estuvo disponible desde el 30-4-21 (confinamiento en España) hasta el 13-5-21 (inicio de la desescalada en España). Los criterios de inclusión fueron el país de residencia (España) y tener más de 50 años. Como criterios de exclusión se asumió la incapacidad para rellenar el cuestionario. Todos los participantes emitieron un consentimiento informado y la aceptación de protección de datos.

Respecto a los instrumentos utilizados, se elaboró un cuestionario de sociodemográficos con algunas preguntas específicas sobre el COVID-19. Además, se construyó un instrumento ad-hoc para la valoración de la realización de las AVD, para ello nos basamos en la taxonomía de actividades de la vida diaria de la Clasificación Internacional de la Salud, la Discapacidad y el Funcionamiento (CIF) de la Organización Mundial de la Salud (OMS, 2001), el índice de Barthel (Mahoney y Barthel, 1975) y la escala de AIVD de Lawton y Brody (1969). De estos instrumentos se extraen una serie de actividades de la vida diaria y con la ayuda de profesionales se llega a una redacción de consenso del contenido del ítem. En total se acuerda introducir 23 ítems, siete recogiendo actividades básicas, cuatro actividades instrumentales y 12 de actividades avanzadas. La idea es conseguir una medida general de mantenimiento de actividad, y dos más específicas, una de actividades necesarias (ABVD y AIVD) y otra de actividades no necesarias (AAVD). Por último, se utilizó la subescala de depresión del GADS 


\section{LAS ACTIVIDADES DE LA VIDA DIARIA EN PERSONAS MAYORES DURANTE EL CONFINAMIENTO}

(Goldberg et al., 1988) en su versión en español usada por Reivan-Ortiz et al. (2019). Este instrumento ha obtenido una fiabilidad de .80 en la subescala de depresión (Reivan-Ortiz et al., 2019), en cuanto a su validez se han encontrado correlaciones entre medias y altas con las dimensiones de un instrumento similar (Lobos-Rivera y Gutiérrez-Quintanilla, 2020)

Respecto a los análisis se han llevado a cabo análisis descriptivos, exploratorios y de correlación, para ello se ha utilizado el paquete estadístico SPSS Statistics versión 25.

\section{RESULTADOS}

\section{Sociodemográficos}

Participaron 418 personas con edades comprendidas entre los 50 y los 90 años. La media de edad fue de 58,51 años. El 67\% fueron mujeres. Y un 82\% vivían acompañados/as. Siendo la mayoría personas casadas (67\%). En la tabla 1 se observan el resto de sociodemográficos.

Tabla 1. Resto sociodemográficos

\begin{tabular}{lll}
\hline Variable & Niveles & Porcentaje \\
\hline Nivel de estudios & Primarios & 13,7 \\
& Secundarios & 29,4 \\
& Universitarios & 55,7 \\
& Sin estudios & 1,3 \\
& & \\
Vive con & Solo & 17,5 \\
& Acompañados & 82,5 \\
Vive en & Casa o chalet & 32 \\
& Piso o & 68 \\
Personas en la & apartamento & \\
vivienda & Una & 17,5 \\
& & \\
& Dos & 37,9 \\
& De tres a cinco & 43 \\
& Seis o más & 1,5 \\
\hline
\end{tabular}

Respecto a las preguntas relacionadas con el COVID y el confinamiento, un 78,6\% percibía durante el confinamiento su que su estado de salud física era el mismo que antes de la cuarentena, frente a un 15,7\% que consideraba que era peor y un 5,7\% que era mejor. En cuanto a estado de ánimo un 54,6\% afirman que era el mismo que antes de la cuarentena frente a un $37,1 \%$ que indicaba que era peor y un $8,2 \%$ que indicaba que era mejor. Y respecto a sus capacidades cognitivas un $81,4 \%$ las percibían igual que antes de la cuarentena frente a un 14,2 que consideraba que eran peores y un 4,4 que consideraba que eran mejores.

La mayoría no había tenido un contacto cercano con individuo con infección confirmada (93,6\%), ni indirecto confirmado (80,9\%). Cerca de un 19,8 había sufrido la pérdida de un familiar o amigo debido al COVID-19

\section{Actividades de la vida diaria}

En la tabla 2, 3 y 4 se muestran los porcentajes de aumento ("Io hago en el confinamiento más que lo hacía antes"), disminución ("Io hago en el confinamiento menos que lo hacia") 0 inalterabilidad de las actividades de la vida diaria de la muestra ("igual que antes"). 
Tabla 2. Realización actividades básicas vida diaria durante confinamiento/ cuarentena.

\begin{tabular}{lllll}
\hline Actividad & Menos & Igual & Más & $\begin{array}{l}\text { Ni lo } \\
\text { hago ni lo } \\
\text { hacia }\end{array}$ \\
\hline $\begin{array}{l}\text { Vestido y } \\
\text { arreglo }\end{array}$ & 50,8 & 47,7 & 0,5 & 1 \\
$\begin{array}{l}\text { Rutina } \\
\text { horaria }\end{array}$ & 20,9 & 69,8 & 4,6 & 4,6 \\
$\begin{array}{l}\text { alimentación } \\
\begin{array}{l}\text { Dieta } \\
\text { adecuada }\end{array}\end{array}$ & 17,3 & 62,1 & 11,9 & 8,8 \\
$\begin{array}{l}\text { Aseo } \\
\text { personal }\end{array}$ & 17 & 75,3 & 7,2 & 0,5 \\
$\begin{array}{l}\text { Deseo sexual } \\
\text { Siesta }\end{array}$ & 21,6 & 59,8 & 4,1 & 14,4 \\
$\begin{array}{l}\text { Rutina de } \\
\text { sueño }\end{array}$ & 11,1 & 44,1 & 19,3 & 25,5 \\
\hline
\end{tabular}

Respecto a las ABVD, se observa que la actividad más afectada negativamente ha sido el vestido y arreglo, así como la más favorecida ha sido la siesta.

Tabla 3. Realización actividades básicas vida diaria durante confinamiento/ cuarentena.

\begin{tabular}{lllll}
\hline Actividad & Menos & Igual & Más & $\begin{array}{l}\text { Ni lo } \\
\text { hago ni lo } \\
\text { hacia }\end{array}$ \\
\hline Tareas hogar & 3,4 & 43 & 52,3 & 1,3 \\
$\begin{array}{l}\text { Hacer la } \\
\text { compra * }\end{array}$ & 45,9 & 33 & 15,2 & 5,9 \\
$\begin{array}{l}\text { Gestión } \\
\begin{array}{l}\text { Económica } \\
\text { Medicación }\end{array}\end{array}$ & 18,3 & 70,6 & 5,4 & 5,7 \\
* se incluye vía telefónica o internet. & 79,6 & 1,5 & 17,8 \\
\hline
\end{tabular}

Dentro de las AIVD la más afectada negativamente ha sido la realización de la compra, y de forma positiva se ha producido un considerable aumento de las tareas del hogar. 


\section{LAS ACTIVIDADES DE LA VIDA DIARIA EN PERSONAS MAYORES DURANTE EL CONFINAMIENTO}

Tabla 4. Realización actividades avanzadas vida diaria durante confinamiento/ cuarentena.

\begin{tabular}{lllll}
\hline Actividad & Menos & Igual & Más & $\begin{array}{l}\text { Ni lo hago } \\
\text { ni lo hacia }\end{array}$ \\
\hline Formación & 16,5 & 31,2 & 14,9 & 37,4 \\
Autoaprendizaje & 3,6 & 46,6 & 40,2 & 9,5 \\
Voluntariado & 8,2 & 22,2 & 3,9 & 65,7 \\
Act. laboral & 23,7 & 41,2 & 8,8 & 26,3 \\
Juego mesa & 9 & 23,7 & 24 & 42,3 \\
TV & 11,6 & 50,5 & 35,6 & 2,3 \\
Radio & 40,7 & 53,9 & 2,8 & 2,6 \\
Leer & 14,4 & 38,9 & 22,7 & 24 \\
Actividad física & 46,1 & 26,5 & 16,2 & 11,1 \\
Contacto & 8,5 & 37,6 & 53,4 & 0,5 \\
familiar & & & & \\
Contacto & 16,5 & 50,5 & 32 & 1 \\
amistad & & & & \\
Contacto social & 23,5 & 42,3 & 9,8 & 24,5 \\
\hline
\end{tabular}

Dentro de las AAVD la más afectada negativamente fue la actividad física, y la que más aumentó fue el contacto familiar.

\section{Estado de ánimo, AVD y edad durante el confinamiento.}

Los niveles de sintomatología depresiva teniendo en cuenta que el máximo era 9 , fueron bajos ( $M=2.1, D T$ $=2,087)$. En lo que respecta a la relación de la sintomatología depresiva con la edad, se encuentra una relación negativa ( $r=-.106, p=.05$ ), es decir, menor sintomatología depresiva a mayor edad. En cuanto a sintomatología depresiva y AVD, si se considera el nivel de actividad total (suma de todas), no se encuentra relación, si nos fijamos en los distintos tipos de AVD, dentro de las ABVD si que se encuentra una relación negativa $(r=-.233, p<$ .01), por tanto, una mayor realización de ABVD tiene relación con una menor sintomatología depresiva. Por último, en cuanto a la relación de las AVD con la edad, se ha encontrado correlación significativa entre la edad y la realización de ABVD ( $r=.181, p=.006)$, encontrándose una relación entre tener más edad y realizar actividades vida diaria. De forma contraria, se ha encontrado una relación negativa entre la edad y las AIVD ( $r=-.141, p=$ .017).

\section{DISCUSIÓN}

Con la edad, el mantenimiento de las AVD se convierte en un aspecto determinante para mantener una buena salud, emocionalidad y calidad de vida (Aravena, 2017; Rubio et al., 2009; Veiguela et al., 2018). El confinamiento provocado por el COVID-19 supuso un punto de inflexión en las rutinas y vidas de las personas. Afectando a las AVD. Este estudio muestra que las actividades que más se vieron afectadas negativamente fueron las ABVD, que se realizaron en menor medida que antes, mientras que las AIVD son las que más aumentaron, seguidas de las AAVD. Las ABVD son aquellas relacionadas con aspectos rutinarios, por tanto, son finalistas y están muy relacionadas con el autocuidado, mientras que a AIVD y AAVD están más relacionadas con el tiempo libre el ocio, su realización es más ocasional, de forma que estas aumentaron al tener más tiempo disponible, pero no se relacionan con aspectos directos de autocuidado como las ABVD (Romero, 2007), indirectamente esto significa que en esta situación de confinamiento los sujetos se centraron más en la distracción/entretenimiento que en aspectos más básicos del autocuidado. No obstante, este estudio ha mostrado una relación entre ABVD y edad, lo que significaría que las personas más mayores si que han mantenido esas conductas de autocuidado y esas actividades rutinarias en mayor medida. 
Por otro lado, pese a que con la edad se aumentaba el riesgo de padecer los efectos nocivos de la infección por COVID-19, Ios niveles de sintomatología depresiva encontrados en este trabajo en personas de mediana edad y mayores, son bajos. Estos resultados van en la línea de otros trabajos similares (Fernández-Ballesteros y Sánchez-Izquierdo, 2020). Además, en este trabajo se ha encontrado una relación entre tener mayor edad y menor sintomatología depresiva, una posible explicación a esto podría ser precisamente el mantenimiento en mayor medida de las ABVD que se ha encontrado en este trabajo. Aspecto que debería ser explorado en profundidad en futuros estudios.

\section{CONCLUSIONES}

Las AVD se han visto afectadas durante el confinamiento en personas de mediana y edad avanzada, especialmente las ABVD. Además, se ha encontrado una relación entre mantener las ABVD y un mejor estado anímico, así como una relación entre edad y mantener ese tipo de actividades.

\section{REFERENCIAS BIBLIOGRÁFICAS}

Aguilar-Parra, J., Lopez-Liria, R., Fernandez-Batanero, J., Padilla-Gongora, D., \& Alvarez, J. (2016). Influencia de la ocupacion en actividades de la vida diaria y de ocio sobre la salud real de la persona mayor. Psychology, Society \& Education, 8(2), 135-147. http://dx.doi.org/10.25115/psye.v8i2.554

Álvarez, P., Scharovsky, D., Brugger, R., Ayala, M., Aleman, A., Sánchez, M., Gonorazky, S., \& Latini, M. (2011). Correlación entre las actividades de la vida diaria y los tests de detección de demencia en nuestra población. Neurología Argentina, 4(3), 112-117. https://doi.org/10.1016/j.neuarg.2012.03.007

Aravena, J. M. (2017). Comprendiendo el impacto de los síntomas depresivos en la funcionalidad de las personas mayores. Revista chilena de neuro-psiquiatría, 55(4), 255-265. http://dx.doi.org/10.4067/s071792272017000400255

Costa, E. C., Nakatani, A. Y. K., \& Bachion, M. M. (2006). Capacidad de los ancianos de una da comunidad en desenvlover Actividades de Vida Diaria y Actividades Instrumentales de Vida Diaria. Acta paulista de enfermagem, 19(1), 43-48.

Escobar, M. Á., Puga, M., y Martín Baranera, M. (2012). Análisis de la esperanza de vida libre de discapacidad a lo largo de la biografía: de la madurez a la vejez. Gaceta Sanitaria, 26(4), 330-335. https://doi.org/10.1016/j.gaceta.2011.12.016

Fernández-Ballesteros, R. y Sánchez-Izquierdo Alonso, M. (2020). Impacto del COVID-19 en personas mayores en España: algunos resultados

Goldberg, D., Bridges, K., Duncan-Jones, P., \& Grayson, D. (1988). Detecting anxiety and depression in general medical settings. British Medical Journal, 297(6653), 897-899. https://doi.org/10.1136/bmj.297.6653.897

Lawton, M.P., Brody, E.M. (1969). Assessment of older people: self-maintaining and instrumental activities of daily living. Gerontologist, 9(3):179-86. https://doi.org/10.1093/geront/9.3_Part_1.179

Lobos-Rivera, M. E., \& Gutiérrez-Quintanilla, J. R. (2020). Adaptación psicométrica de la escala de ansiedad y depresión de Goldberg en una muestra salvadoreña. Entorno, 70, 87-98. https://doi.org/10.5377/entorno.v0i69.9557

Losada-Baltar, A., Márquez-González, M., Jiménez-Gonzalo, L., Pedroso-Chaparro, M., Gallego-Alberto, L., \& Fernandes-Pires, J. (2020). Diferencias en función de la edad y la autopercepción del envejecimiento en ansiedad, tristeza, soledad y sintomatología comórbida ansioso-depresiva durante el confinamiento por la COVID-19. Revista española de geriatría y gerontología, 55(5), 272-278. https://doi.org/10.1016/j.regg.2020.05.005

Meléndez, J. C., Tomás, J. M. y Navarro, E. (2011). Actividades de la vida diaria y bienestar y su relación con la edad y el género en la vejez. Anales De Psicología, 27(1), 164-169. https://doi.org/10.6018/analesp

Reivan-Ortiz, G., Pineda-Garcia, G., \& León Parias, B. D. (2019). Psychometric properties of the goldberg anxiety 


\section{LAS ACTIVIDADES DE LA VIDA DIARIA EN PERSONAS MAYORES DURANTE EL CONFINAMIENTO}

and depression scale (GADS) in ecuadorian population. International Journal of Psychological Research, 12(1), 41-48. https://doi.org/10.21500/20112084.3745

Romero, D.M.(2007).Actividad vida diaria .Anales De Psicología, 23(2), 264. https://www.proquest.com/scholarly-journals/actividades-de-la-vida-diaria/docview/1288744099/se-2?accountid=14777

Rubio, E., Lázaro, A., Martínez, T., y Magallón, R. (2009). Enfermedades crónicas y deterioro funcional para las actividades de la vida diaria en población mayor no institucionalizada. Revista española de geriatría y gerontología, 44(5), 244-250. https://doi.org/10.1016/j.regg.2009.03.011

Veiguela, D. R., Álvarez, A. Á., Loureda, R. A., Cela, M. V., \& Celeiro, I. D. R. (2018). Explorando las actividades diarias y la participación social: Un estudio en personas con una condición de salud del aparato locomotor. Cadernos De Terapia Ocupacional Da UFSCar, 26(3), 513-526. doi:http://dx.doi.org/10.4322/25268910.ctoA01661 y reflexiones. Clínica y Salud, 31(3), 165-169. https://doi.org/10.5093/clysa2020a25 